\title{
ON $m$-ACCRETIVE SCHRÖDINGER-TYPE OPERATORS WITH SINGULAR POTENTIALS ON MANIFOLDS OF BOUNDED GEOMETRY
}

\author{
OGNJEN MILATOVIC
}

Received 20 September 2002

\begin{abstract}
We consider a Schrödinger-type differential expression $\nabla^{*} \nabla+V$, where $\nabla$ is a $C^{\infty}$-bounded Hermitian connection on a Hermitian vector bundle $E$ of bounded geometry over a manifold of bounded geometry $(M, g)$ with positive $C^{\infty}$-bounded measure $d \mu$, and $V$ is a locally integrable linear bundle endomorphism. We define a realization of $\nabla^{*} \nabla+V$ in $L^{2}(E)$ and give a sufficient condition for its $m$ accretiveness. The proof essentially follows the scheme of T. Kato, but it requires the use of a more general version of Kato's inequality for Bochner Laplacian operator as well as a result on the positivity of solution to a certain differential equation on $M$.
\end{abstract}

2000 Mathematics Subject Classification: 35P05, 58J50, 47B25, 81Q10.

\section{Introduction and the main result}

1.1. The setting. Let $(M, g)$ be a $C^{\infty}$ Riemannian manifold without boundary, with metric $g, \operatorname{dim} M=n$. We will assume that $M$ is connected. We will also assume that $M$ has bounded geometry. Moreover, we will assume that we are given a positive $C^{\infty}$-bounded measure $d \mu$, that is, in any local coordinates $x^{1}, x^{2}, \ldots, x^{n}$, there exists a strictly positive $C^{\infty}$-bounded density $\rho(x)$ such that $d \mu=\rho(x) d x^{1} d x^{2} \cdots d x^{n}$.

Let $E$ be a Hermitian vector bundle over $M$. We will assume that $E$ is a bundle of bounded geometry (i.e., it is supplied by an additional structure: trivializations of $E$ on every canonical coordinate neighborhood $U$ such that the corresponding matrix transition functions $h_{U, U^{\prime}}$ on all intersections $U \cap U^{\prime}$ of such neighborhoods are $C^{\infty}$-bounded, that is, all derivatives $\partial_{y}^{\alpha} h_{U, U^{\prime}}(y)$, where $\alpha$ is a multiindex, with respect to canonical coordinates, are bounded with bounds $C_{\alpha}$ which do not depend on the chosen pair $U, U^{\prime}$ ).

We denote by $L^{2}(E)$ the Hilbert space of square integrable sections of $E$ with respect to the scalar product

$$
(u, v)=\int_{M}\langle u(x), v(x)\rangle_{E_{x}} d \mu(x)
$$

Here $\langle\cdot, \cdot\rangle_{E_{x}}$ denotes the fiberwise inner product. 
Let

$$
\nabla: C^{\infty}(E) \longrightarrow C^{\infty}\left(T^{*} M \otimes E\right)
$$

be a Hermitian connection on $E$ which is $C^{\infty}$-bounded as a linear differential operator, that is, in any canonical coordinate system $U$ (with the chosen trivializations of $\left.E\right|_{U}$ and $\left.\left.\left(T^{*} M \otimes E\right)\right|_{U}\right), \nabla$ is written in the form

$$
\nabla=\sum_{|\alpha| \leq 1} a_{\alpha}(y) \partial_{y}^{\alpha}
$$

where $\alpha$ is a multiindex, and the coefficients $a_{\alpha}(y)$ are matrix functions whose derivatives $\partial_{y}^{\beta} a_{\alpha}(y)$ for any multiindex $\beta$ are bounded by a constant $C_{\beta}$ which does not depend on the chosen canonical neighborhood.

We will consider a Schrödinger type differential expression of the form

$$
H_{V}=\nabla^{*} \nabla+V
$$

Here

$$
\nabla^{*}: C^{\infty}\left(T^{*} M \otimes E\right) \longrightarrow C^{\infty}(E)
$$

is a differential operator which is formally adjoint to $\nabla$ with respect to the scalar product (1.1), and $V$ is a linear bundle endomorphism of $E$, that is, for every $x \in M$,

$$
V(x): E_{x} \longrightarrow E_{x}
$$

is a linear operator.

We make the following assumption on $V$.

Assumption 1.1. Assume that $V \in L_{\mathrm{loc}}^{p}($ End $E)$, where

(i) $p=2 n /(n+2)$ for $n \geq 3$,

(ii) $p>1$ for $n=2$,

(iii) $p=1$ for $n=1$.

We will use the following notations:

$$
V_{1}(x):=\frac{V(x)+(V(x))^{*}}{2}, \quad V_{2}(x):=\frac{V(x)-(V(x))^{*}}{2 i}, \quad x \in M,
$$

where $i=\sqrt{-1}$ and $(V(x))^{*}$ denotes the adjoint of the linear operator (1.6) (in the sense of linear algebra).

By (1.7), for all $x \in M$, we have the following decomposition:

$$
V(x)=V_{1}(x)+i V_{2}(x) .
$$


1.2. Sobolev space $W^{1,2}(E)$. By $W^{1,2}(E)$ we will denote the set of all $u \in$ $L^{2}(E)$ such that $\nabla u \in L^{2}\left(T^{*} M \otimes E\right)$. It is well known (see, e.g., [5, Section A1.1]) that $W^{1,2}(E)$ is the completion of the space $C_{C}^{\infty}(E)$ with respect to the norm $\|\cdot\|_{1}$ defined by the scalar product

$$
(u, v)_{1}:=(u, v)+(\nabla u, \nabla v), \quad u, v \in C_{c}^{\infty}(E)
$$

By $W^{-1,2}(E)$ we will denote the dual of $W^{1,2}(E)$.

Since $(M, g)$ and $E$ have bounded geometry, by [5, Section A1.1], it follows that the usual Sobolev embedding theorem (see, e.g., [1, Theorem 2.21]) holds for $W^{1,2}(E)$.

1.3. A realization of $H_{V}$ in $L^{2}(E)$. Let $V$ be as in Assumption 1.1. We define an operator $S$ associated to $H_{V}$ as an operator in $L^{2}(E)$ given by $S u=H_{V} u$ with domain

$$
\operatorname{Dom}(S)=\left\{u \in W^{1,2}(E): H_{V} u \in L^{2}(E)\right\}
$$

REMARK 1.2. We will show that for all $u \in W^{1,2}(E)$, we have $V u \in L_{\text {loc }}^{1}(E)$ so that $H_{V} u$ in (1.10) can be understood in distributional sense.

Let $u \in W^{1,2}(E)$. For $n \geq 3$, by Section 1.2 above and the first part of Theorem 2.21 from Aubin [1], we have the following continuous embedding

$$
W^{1,2}(E) \subset L^{p^{\prime}}(E),
$$

where $1 / p^{\prime}=1 / 2-1 / n$.

Let $p=2 n /(n+2)$ be as in Assumption 1.1. Since $1 / p+1 / p^{\prime}=1$, by Hölder's inequality, it follows that $V u \in L_{\text {loc }}^{1}(E)$.

For $n=2$, by the first part of Theorem 2.21 from Aubin [1], we get the continuous embedding (1.11) for all $2<p^{\prime}<\infty$. By Assumption 1.1, for $n=2$, we have $p>1$. We may assume that $1<p<2$ (if $V \in L_{\text {loc }}^{t}($ End $E$ ) with $t \geq 2$, then $V \in L_{\mathrm{loc}}^{p}\left(\right.$ End $E$ ) for all $1<p<2$ ). Given $1<p<2$, we can take $p^{\prime}>2$ such that $1 / p+1 / p^{\prime}=1$. By Hölder's inequality, we have $V u \in L_{\mathrm{loc}}^{1}(E)$.

For $n=1$, it is well known (see, e.g., the second part of Theorem 2.21 in [1]) that (1.11) holds with $p^{\prime}=\infty$. By Assumption 1.1, for $n=1$, we have $p=1$. Thus, by Hölder's inequality, we have $V u \in L_{\text {loc }}^{1}(E)$.

We now state the main result.

THEOREM 1.3. Assume that $(M, g)$ is a manifold of bounded geometry with a positive $C^{\infty}$-bounded measure $d \mu$. Assume that $E$ is a Hermitian vector bundle of bounded geometry over $M$. Assume $\nabla$ to be a $C^{\infty}$-bounded Hermitian connection 
on E. Let $V$ be as in Assumption 1.1. Moreover, assume that for all $x \in M$,

$$
V_{1}(x) \geq 0, \quad \text { as an operator } E_{x} \rightarrow E_{x},
$$

where $V_{1}(x)$ is as in (1.7).

Then $S$ is $m$-accretive.

REMARK 1.4. The main source of inspiration for Theorem 1.3 was a result of Kato [3, Theorem I] which was proven for the operator $-\Delta+V$ on an open set $\Omega \subset \mathbb{R}^{n}$, where $-\Delta$ is the standard Laplacian on $\mathbb{R}^{n}$ with the standard metric and measure, and $V \in L_{\mathrm{loc}}^{p}(\Omega)$, with $p$ as in Assumption 1.1, is a complexvalued function such that $\operatorname{Re} V \geq 0$.

Let $d: C^{\infty}(M) \rightarrow \Omega^{1}(M)$ be the standard differential. Then $d^{*} d: C^{\infty}(M) \rightarrow$ $C^{\infty}(M)$ is called the scalar Laplacian and will be denoted by $\Delta_{M}$.

2. Proof of Theorem 1.3. We will adopt the proof of [3, Theorem I] in our context. Throughout this section, we assume that all hypotheses of Theorem 1.3 are satisfied. We begin by introducing another realization of $H_{V}$.

2.1. Maximal realization of $H_{V}$ between $W^{1,2}(E)$ and $W^{-1,2}(E)$. We define an operator $T$ associated to $H_{V}$ as an operator $W^{1,2}(E) \rightarrow W^{-1,2}(E)$ given by $T u=H_{V} u$ with domain

$$
\operatorname{Dom}(T)=\left\{u \in W^{1,2}(E): H_{V} u \in W^{-1,2}(E)\right\} .
$$

REMARK 2.1. Condition $H_{V} u \in W^{-1,2}(E)$ for $u \in W^{1,2}(E)$ makes sense since $H_{V} u$ is a distributional section of $E$ by Remark 1.2. Since $\nabla^{*} \nabla u \in W^{-1,2}(E)$ for $u \in W^{1,2}(E)$, it follows that the condition $H_{V} u \in W^{-1,2}(E)$ in (2.1) is equivalent to $V u \in W^{-1,2}(E)$ for $u \in W^{1,2}(E)$.

LEMMA 2.2. The following inclusion holds: $C_{c}^{\infty}(E) \subset \operatorname{Dom}(T)$.

Proof. Let $u \in C_{c}^{\infty}(E)$. Then $V u \in L^{p}(E)$, where $p$ is as in Assumption 1.1. By Remark 1.2, it follows that $W^{1,2}(E) \subset L^{p^{\prime}}(E)$, where $1 / p+1 / p^{\prime}=1$. By duality, we have $L^{p}(E) \subset W^{-1,2}(E)$. Thus $V u \in W^{-1,2}(E)$, and hence $u \in \operatorname{Dom}(T)$.

2.2. Minimal realization of $H_{V}$ between $W^{1,2}(E)$ and $W^{-1,2}(E)$. By $T_{0}$ we will denote the restriction of $T$ with $\operatorname{Dom}\left(T_{0}\right)=C_{c}^{\infty}(E)$. Clearly, $T_{0}$ is a densely defined operator.

REMARK 2.3. Since $\operatorname{Dom}(S)$, where $S$ is as in (1.10), does not necessarily contain $C_{C}^{\infty}(E)$, there is no minimal realization of $H_{V}$ in $L^{2}(E)$ (in the sense of Section 2.2).

2.3. Maximal and minimal realization of $H_{V^{*}}$. In what follows, we will denote by $T^{\prime}$ and $T_{0}^{\prime}$ the maximal and minimal realization of $H_{V^{*}}$ in the sense of Sections 2.1 and 2.2, respectively, where $V^{*}$ is the adjoint of $V$ as in (1.7). 
LEMMA 2.4. The following holds: $T=\left(T_{0}^{\prime}\right) *$, where $*$ denotes the adjoint of an operator.

Proof. We need to show that for any $u \in W^{1,2}(E)$ and $f \in W^{-1,2}(E)$, the equation $T u=f$ is true if and only if

$$
\left(u, T^{\prime} s\right)=(f, s), \quad \forall s \in C_{c}^{\infty}(E),
$$

where $(\cdot, \cdot)$ denotes the duality between $W_{\text {loc }}^{1,2}(E)$ and $W_{\text {comp }}^{-1,2}(E)$ extending the inner product in $L^{2}(E)$ by continuity from $C_{C}^{\infty}(E)$.

(1) Assume that $u \in W^{1,2}(E), f \in W^{-1,2}(E)$, and $T u=f$. Then $V u \in$ $W^{-1,2}(E)$. By Lemma 2.2, for all $s \in C_{c}^{\infty}(E)$, we have $V^{*} s \in W_{\text {comp }}^{-1,2}(E)$. Since $s \in C_{c}^{\infty}(E)$, we have $V^{*} s \in L_{\text {comp }}^{p}(E)$ with $p$ as in Assumption 1.1. By the proof in Remark 1.2, we have $u \in W^{1,2}(E) \subset L^{p^{\prime}}(E)$ (continuous embedding), where $1 / p+1 / p^{\prime}=1$. By Hölder's inequality, $L_{\mathrm{loc}}^{p^{\prime}}(E)$ is in a continuous duality with $L_{\text {comp }}^{p}(E)$ by the usual integration. Thus, for all $s \in C_{c}^{\infty}(E)$, we have (after approximating $u$ by sections $u_{j} \in C_{C}^{\infty}(E)$ in $W^{1,2}$-norm in a neighborhood of supp $s$ )

$$
\begin{aligned}
\left(u, V^{*} s\right) & =\lim _{j \rightarrow \infty}\left(u_{j}, V^{*} s\right)=\lim _{j \rightarrow \infty} \int\left\langle u_{j}(x),\left(V^{*} s\right)(x)\right\rangle d \mu(x) \\
& =\int\left\langle u(x),\left(V^{*} s\right)(x)\right\rangle d \mu(x),
\end{aligned}
$$

where $(\cdot, \cdot)$ is as in (2.2). The second equality in (2.3) holds since $V^{*} s \in L_{\text {loc }}^{1}(E)$ by Remark 1.2 and $u_{j} \in C_{c}^{\infty}(E)$.

Therefore, we obtain

$$
\begin{aligned}
\left(u, V^{*} s\right) & =\int\left\langle u(x),\left(V^{*} s\right)(x)\right\rangle d \mu(x) \\
& =\int\langle(V u)(x), s(x)\rangle d \mu(x)=(V u, s),
\end{aligned}
$$

where $(\cdot, \cdot)$ is as in (2.2). The first equality in (2.4) follows from (2.3). The second equality in (2.4) holds by the definition of $(V(x))^{*}: E_{x} \rightarrow E_{x}$. The third equality in (2.4) holds for all $s \in C_{c}^{\infty}(E)$ since $V u \in W^{-1,2}(E)$ and $V u \in L_{\mathrm{loc}}^{1}(E)$ by Remark 1.2.

Using (2.4), we obtain

$$
\begin{aligned}
\left(u, T^{\prime} s\right) & =\left(u, \nabla^{*} \nabla s+V^{*} s\right)=\left(u, \nabla^{*} \nabla s\right)+\left(u, V^{*} s\right) \\
& =\left(\nabla^{*} \nabla u, s\right)+(V u, s)=(T u, s),
\end{aligned}
$$

where $V^{*}$ is the adjoint of $V$ as in (1.7) and $(\cdot, \cdot)$ is as in (2.2). In the third equality, we also used the integration by parts (see, e.g., [2, Lemma 8.8]).

(2) Assume that $u \in W^{1,2}(E), f \in W^{-1,2}(E)$, and (2.2) holds. Then the first two equalities in (2.4) hold (we do not know a priori that $V u \in W^{-1,2}(E)$ so the 
third equality in (2.4) is not yet justified). Thus for all $s \in C_{c}^{\infty}(E)$,

$$
\begin{aligned}
(f, s) & =\left(u, T^{\prime} s\right)=\left(u, \nabla^{*} \nabla s\right)+\left(u, V^{*} s\right) \\
& =\left(\nabla^{*} \nabla u, s\right)+\int\langle(V u)(x), s(x)\rangle d \mu(x),
\end{aligned}
$$

where the second equality follows as in (2.5), and the third equality follows from integration by parts and the second equality in (2.4).

Since $\nabla^{*} \nabla u \in W^{-1,2}(E)$ and $f \in W^{-1,2}(E)$, we obtain

$$
\left(f-\nabla^{*} \nabla u, s\right)=\int\langle(V u)(x), s(x)\rangle d \mu(x), \quad \forall s \in C_{c}^{\infty}(E),
$$

where $(\cdot, \cdot)$ is as in (2.2).

Since $u \in W^{1,2}(E)$, from Remark 1.2, we know that $V u \in L_{\mathrm{loc}}^{1}(E)$. By (2.7), we get $V u \in W^{-1,2}(E)$ since $C_{c}^{\infty}(E)$ is dense in $W^{1,2}(E)$. Thus, as in (2.4),

$$
\int\langle(V u)(x), s(x)\rangle d \mu(x)=(V u, s), \quad \forall s \in C_{c}^{\infty}(E),
$$

where $(\cdot, \cdot)$ is as in $(2.2)$.

From (2.7) and (2.8), we obtain

$$
\left(f-\nabla^{*} \nabla u, s\right)=(V u, s), \quad \forall s \in C_{c}^{\infty}(E),
$$

where $(\cdot, \cdot)$ is as in (2.2).

Therefore,

$$
(f, s)=\left(\nabla^{*} \nabla u, s\right)+(V u, s)=(T u, s), \quad \forall s \in C_{c}^{\infty}(E),
$$

where $(\cdot, \cdot)$ is as in (2.2).

This shows that $T u=f$, and the lemma is proven.

In what follows, we will adopt the terminology of Kato [3] and distinguish between monotone and accretive operators. Accretive operators act within the same Hilbert space, while monotone operators act from a Hilbert space into its adjoint space (antidual).

LEMMA 2.5. The operator $T_{0}$ is monotone, that is,

$$
\operatorname{Re}\left(T_{0} s, s\right) \geq 0, \quad \forall s \in C_{c}^{\infty}(E),
$$

where $(\cdot, \cdot)$ denotes the duality between $W^{-1,2}(E)$ and $W^{1,2}(E)$.

Proof. We have for all $s \in C_{c}^{\infty}(E)$,

$$
\begin{aligned}
\operatorname{Re}\left(T_{0} s, s\right) & =\operatorname{Re}\left[\left(\nabla^{*} \nabla s, s\right)+\int\langle V s, s\rangle d \mu\right] \\
& =\|\nabla s\|^{2}+\operatorname{Re}\left[\int\left\langle V_{1} s, s\right\rangle d \mu+i \int\left\langle V_{2} s, s\right\rangle d \mu\right] \\
& \geq\|\nabla s\|^{2},
\end{aligned}
$$


where $(\cdot, \cdot)$ is as in (2.11), $\|\cdot\|$ denotes the $L^{2}$-norm, and $V_{1} \geq 0$ and $V_{2}$ are linear selfadjoint bundle endomorphisms as in (1.7).

The lemma is proven.

LEMMA 2.6. The operator $1+T_{0}$ is coercive in the sense that

$$
\left\|\left(1+T_{0}\right) s\right\|_{-1} \geq\|s\|_{1}, \quad \forall s \in \operatorname{Dom}\left(T_{0}\right)=C_{c}^{\infty}(E),
$$

where $\|\cdot\|_{-1}$ is the norm in $W^{-1,2}(E)$, and $\|\cdot\|_{1}$ is the norm in $W^{1,2}(E)$.

Proof. As in (2.12), we have for all $s \in C_{c}^{\infty}(E)$,

$$
\operatorname{Re}\left(\left(T_{0}+1\right) s, s\right) \geq\|s\|^{2}+\|\nabla s\|^{2}=\|s\|_{1}^{2},
$$

where $(\cdot, \cdot)$ is as in $(2.11)$.

Since the left-hand side of (2.14) does not exceed $\left\|\left(1+T_{0}\right) s\right\|_{-1}\|s\|_{1}$, inequality (2.13) immediately follows from (2.14).

In what follows, $\operatorname{Ker} A$ and $\operatorname{Ran} A$ denote the kernel and the range of operator $A$, respectively, and $\bar{A}$ denotes the closure of $A$.

LEMMA 2.7. The following holds:

(i) the operator $T_{0}$ is closable with closure $T_{0}^{* *}$,

(ii) $\operatorname{Ran}\left(1+T_{0}^{* *}\right)$ is closed.

Proof. By Lemma 2.4, it follows that $T^{\prime}=T_{0}^{*}$, where $T^{\prime}$ is as in Section 2.3. Since $T_{0}^{\prime} \subset T^{\prime}$ (as operators), it follows that $T^{\prime}$ is densely defined. Thus $T_{0}^{* *}$ exists and equals $\overline{T_{0}}$. This proves property (i).

We will now prove property (ii). Since $1+T_{0}$ is coercive by Lemma 2.6 , it follows by definition of $\overline{T_{0}}$ that $1+T_{0}^{* *}=1+\overline{T_{0}}$ is also coercive, that is,

$$
\left\|\left(1+T_{0}^{* *}\right) u\right\|_{-1} \geq\|u\|_{1}, \quad \forall u \in \operatorname{Dom}\left(T_{0}^{* *}\right),
$$

where $\|\cdot\|_{-1}$ is the norm in $W^{-1,2}(E)$, and $\|\cdot\|_{1}$ is the norm in $W^{1,2}(E)$.

We will now show that $\operatorname{Ran}\left(1+T_{0}^{* *}\right)$ is closed.

Let $f_{j} \in \operatorname{Ran}\left(1+T_{0}^{* *}\right)$ and $\left\|f_{j}-f\right\|_{-1} \rightarrow 0$ as $j \rightarrow \infty$. Let $u_{j} \in \operatorname{Dom}\left(1+T_{0}^{* *}\right)$ be a sequence such that $\left(1+T_{0}^{* *}\right) u_{j}=f_{j}$. Since $f_{j}$ is a Cauchy sequence in $\|\cdot\|_{-1}$, by (2.15) it follows that $u_{j}$ is a Cauchy sequence in $\|\cdot\|_{1}$. Thus $u_{j}$ converges in $\|\cdot\|_{1}$, and we will denote its limit by $u$. Since $1+T_{0}^{* *}$ is a closed operator, it follows that $u \in \operatorname{Dom}\left(1+T_{0}^{* *}\right)$ and $f=\left(1+T_{0}^{* *}\right) u$. Thus $f \in \operatorname{Ran}\left(1+T_{0}^{* *}\right)$, and property (ii) is proven.

In what follows, we will use the general version of Kato's inequality whose proof is given in [2, Theorem 5.7].

LEMMA 2.8. Assume that $(M, g)$ is a Riemannian manifold. Assume that $E$ is a Hermitian vector bundle over $M$ and $\nabla$ is a Hermitian connection on $E$. 
Assume that $w \in L_{\mathrm{loc}}^{1}(E)$ and $\nabla^{*} \nabla w \in L_{\mathrm{loc}}^{1}(E)$. Then

$$
\Delta_{M}|w| \leq \operatorname{Re}\left\langle\nabla^{*} \nabla w, \operatorname{sign} w\right\rangle,
$$

where $\Delta_{M}$ is the scalar Laplacian on $M$ and

$$
\operatorname{sign} w(x)= \begin{cases}\frac{w(x)}{|w(x)|} & \text { if } w(x) \neq 0 \\ 0 & \text { otherwise. }\end{cases}
$$

We now state and prove the key proposition.

Proposition 2.9. The following holds: $\operatorname{Ran}\left(1+T_{0}^{* *}\right)=W^{-1,2}(E)$.

Proof. By Lemma 2.7, it suffices to show that if $u \in W^{1,2}(E)$ and

$$
\left(\left(1+T_{0}\right) s, u\right)=0, \quad \forall s \in C_{c}^{\infty}(E)
$$

where $(\cdot, \cdot)$ is as in (2.11), then $u=0$.

Using condition (2.18) and the same arguments as in the proof of the first two equalities in (2.4) and the equation (2.8), we have

$$
\begin{aligned}
0 & =(s, u)+\left(\nabla^{*} \nabla s, u\right)+(V s, u) \\
& =(s, u)+\left(s, \nabla^{*} \nabla u\right)+\left(s, V^{*} u\right), \quad \forall s \in C_{c}^{\infty}(E),
\end{aligned}
$$

where $(\cdot, \cdot)$ is as in $(2.11)$, and $V^{*}$ is as in (1.7).

Therefore, the following distributional equality holds (recall that by Remark 1.2 , we have $\left.V^{*} u \in L_{\text {loc }}^{1}(E)\right)$

$$
\nabla^{*} \nabla u+V^{*} u+u=0
$$

From (2.20), we have $\nabla^{*} \nabla u=-V^{*} u-u \in L_{\text {loc }}^{1}(E)$. Therefore, by Lemma 2.8, we get

$$
\begin{aligned}
\Delta_{M}|u| & \leq \operatorname{Re}\left\langle\nabla^{*} \nabla u, \operatorname{sign} u\right\rangle \\
& =\operatorname{Re}\left\langle-u-V_{1} u+i V_{2} u, \operatorname{sign} u\right\rangle \\
& =-|u|-\left\langle V_{1} u, \operatorname{sign} u\right\rangle \leq-|u|,
\end{aligned}
$$

where $\Delta_{M},\langle\cdot, \cdot\rangle$ and $\operatorname{sign} u$ are as in (2.16), and $V_{1} \geq 0, V_{2}$ are linear selfadjoint bundle endomorphisms as in (1.7).

By (2.21), we get the following distributional inequality:

$$
\left(\Delta_{M}+1\right)|u| \leq 0
$$

Since $(M, g)$ is a manifold of bounded geometry, by [2, Proposition B.3], inequality (2.22) implies that $|u|=0$, that is, $u=0$. This concludes the proof of the proposition. 
COROLLARY 2.10. The operator $T_{0}^{* *}$ is maximal monotone (in the sense that it is monotone and has no proper monotone extension).

PROOF. The corollary follows immediately from Proposition 2.9, inequality (2.15), and the remark after equation (3.38) of [4, Section 5.3.10].

Proposition 2.11. The following holds:

(i) $T=T_{0}^{* *}$,

(ii) the operator $T$ is maximal monotone.

Proof. We first prove property (i). Since $T_{0} \subset T$ (as operators), it follows that $T_{0}^{* *} \subset T$ because $T$ is closed by Lemma 2.4. By Proposition 2.9, Ran(1+ $\left.T_{0}^{* *}\right)=W^{-1,2}(E)$. By the same proposition (with $V$ replaced by $V^{*}$ ), it follows that $\operatorname{Ran}\left(1+\left(T_{0}^{\prime}\right) * *\right)=W^{-1,2}(E)$, where $T_{0}^{\prime}$ is as in Section 2.3. Since $1+T=$ $1+\left(T_{0}^{\prime}\right) *$ (see Lemma 2.4), it follows that $\operatorname{Ker}(1+T)=\{0\}$. Hence, $T$ cannot be a proper extension of $T_{0}^{* *}$. This shows that $T_{0}^{* *}=T$.

Property (ii) follows immediately from property (i) and Corollary 2.10.

3. Proof of Theorem 1.3. First, note that the following holds: $u \in \operatorname{Dom}(S)$ if and only if $u \in \operatorname{Dom}(T)$ and $T u \in L^{2}(E)$ (in which case $S u=T u$ ).

By Propositions 2.9 and 2.11, it follows that $\operatorname{Ran}(1+T)=W^{-1,2}(E)$. Therefore, $\operatorname{Ran}(1+S)=L^{2}(E)$. Furthermore, since $T$ is maximal monotone by Proposition 2.11, it follows that

$$
\operatorname{Re}(S u, u)_{L^{2}(E)}=\operatorname{Re}(T u, u) \geq 0, \quad \forall u \in \operatorname{Dom}(S),
$$

where $(\cdot, \cdot)_{L^{2}(E)}$ denotes the inner product in $L^{2}(E)$, and $(\cdot, \cdot)$ is the duality between $W^{-1,2}(E)$ and $W^{1,2}(E)$.

Thus we proved that $S$ is accretive and $\operatorname{Ran}(1+S)=L^{2}(E)$. By the remark after equation (3.37) of [4, Section 5.3.10], it follows that $S$ is $m$-accretive.

\section{REFERENCES}

[1] T. Aubin, Some Nonlinear Problems in Riemannian Geometry, Springer Monographs in Mathematics, Springer-Verlag, Berlin, 1998.

[2] M. Braverman, O. Milatovic, and M. Shubin, Essential self-adjointness of Schrödinger-type operators on manifolds, Russian Math. Surveys 57 (2002), no. 4, 641-692.

[3] T. Kato, On some Schrödinger operators with a singular complex potential, Ann. Scuola Norm. Sup. Pisa Cl. Sci. (4) 5 (1978), no. 1, 105-114.

[4] _ Perturbation Theory for Linear Operators, Springer-Verlag, New York, 1980.

[5] M. A. Shubin, Spectral theory of elliptic operators on noncompact manifolds, Astérisque (1992), no. 207, 35-108.

Ognjen Milatovic: Department of Mathematics, Fitchburg State College, 160 Pearl Street, Fitchburg, MA 01420, USA

E-mail address: omi 1atovic@fsc.edu 


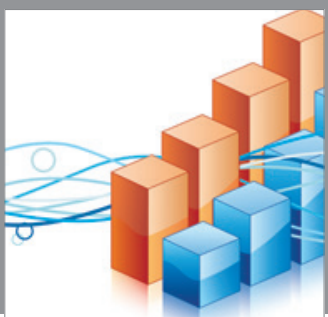

Advances in

Operations Research

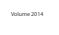

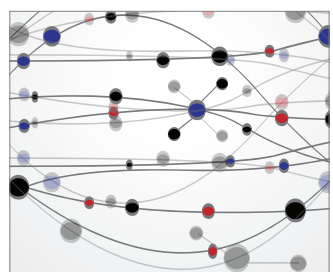

\section{The Scientific} World Journal
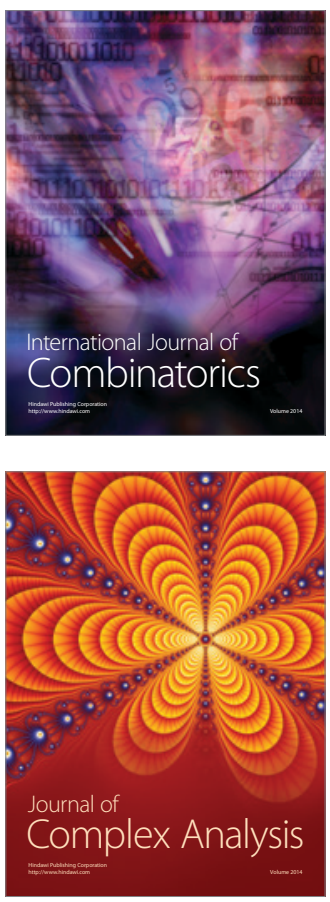

International Journal of

Mathematics and

Mathematical

Sciences
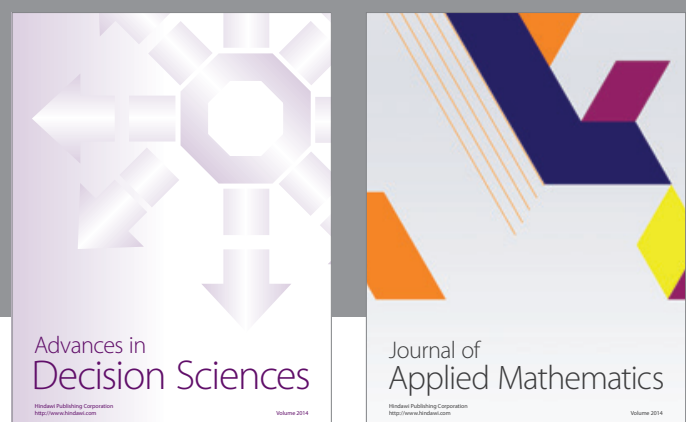

Journal of

Applied Mathematics
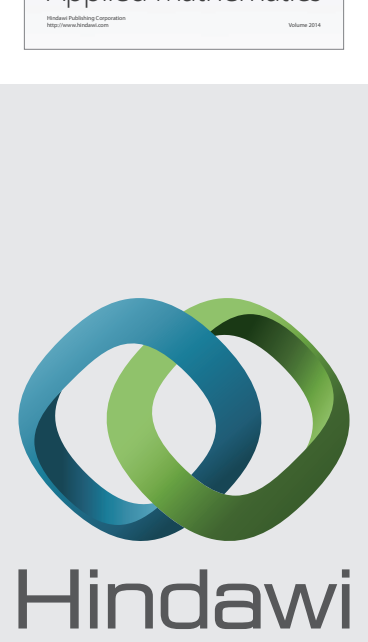

Submit your manuscripts at http://www.hindawi.com
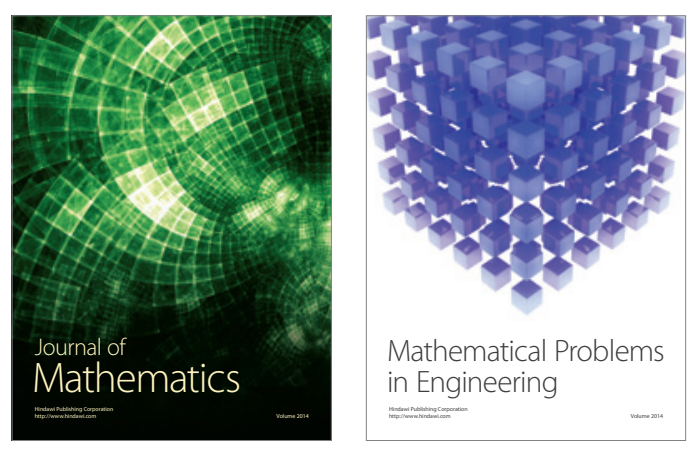

Mathematical Problems in Engineering
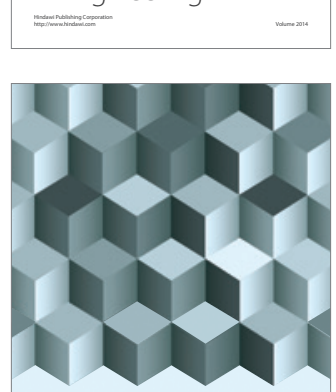

Journal of

Function Spaces
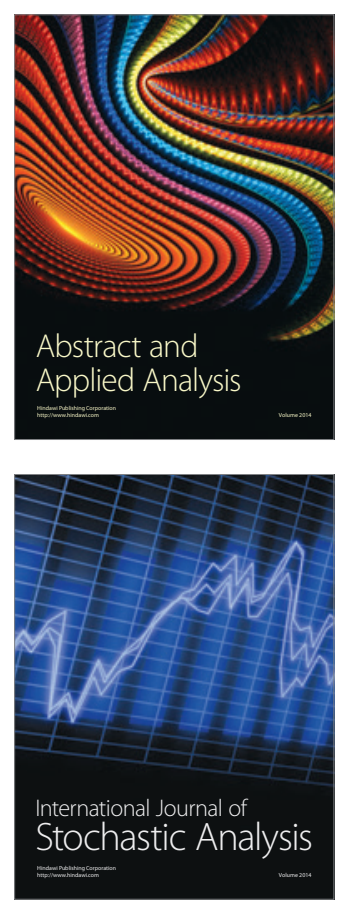

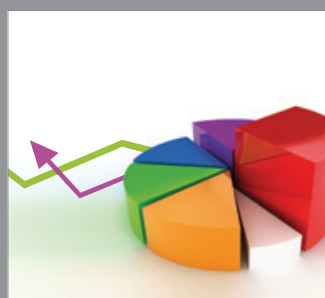

ournal of

Probability and Statistics

Promensencen
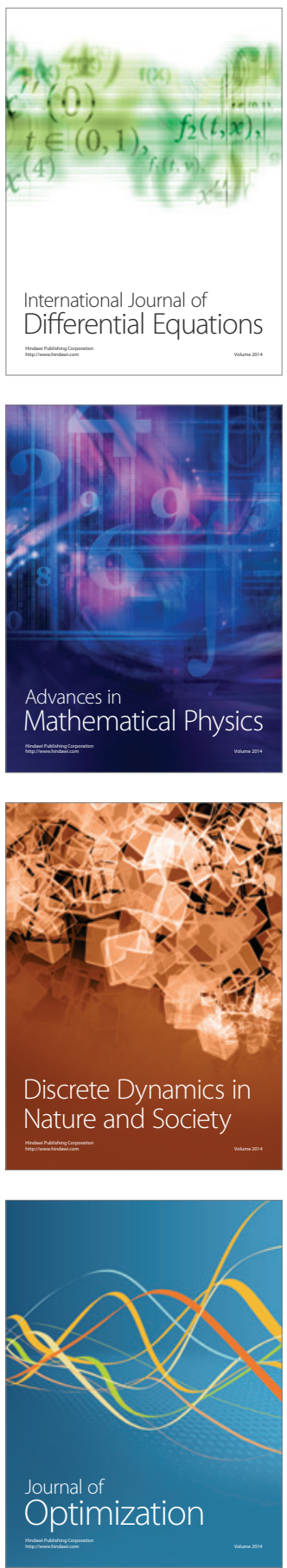